\title{
Corneal ulceration in the elderly in Hyderabad, south India
} Derek Y Kunimoto, Savitri Sharma, Prashant Garg, Usha Gopinathan, David Miller,
Gullapalli N Rao
Harvard Medical School, Department of Ophthalmology, Boston, MA, USA D Y Kunimoto D Miller

LV Prasad Eye Institute, Hyderabad, India

S Sharma

P Garg

U Gopinathan

G N Rao

Correspondence to: Savitri Sharma, LV Prasad Eye Institute, Road No 2, Bajara Hills, Hyderabad 500 034, India

Accepted for publication 18 August 1999

\begin{abstract}
Aims-To report demographic, microbiological, therapeutic, anatomical, and visual results of corneal ulceration in the elderly patients seen at a tertiary eye care centre in south India.

Methods-102 consecutive cases of microbial keratitis in patients 65 years and older were studied. Inclusion criteria were: (i) presence of corneal stromal infiltrate upon slit lamp examination; and (ii) microbiological evaluation of corneal scrapings for suspected microbial keratitis.

Results-The principal predisposing factors identified in this study were ocular disease $(38.2 \%)$, previous ocular surgery in the same eye $(29.4 \%)$, trauma $(17.6 \%)$, and severe systemic disease $(16.7 \%)$. Contact lens wear was associated with only two cases $(2.0 \%) .99$ organisms were isolated in cultures of corneal scrapings from 74 $(72.5 \%)$ of the 102 cases. Staphylococcus epidermidis (31.1\%), filamentous fungi (25.7\%), and Streptococcus pneumoniae $(13.5 \%)$ were the most common isolates. 12 eyes $(11.8 \%)$ required surgery, 15 $(14.7 \%)$ eventually required evisceration, and nine $(9.6 \%)$ of the 94 followed patients achieved an unaided vision of $20 / 60$ or better at last follow up.

Conclusions-This work represents the largest recent single centre study on (nonviral) microbial keratitis in the elderly, its management, and outcomes of therapy. While the predisposing factors differ from those of general population, the spectrum of microbes responsible for keratitis in the elderly appears to reflect the local microbial flora rather than a predilection for elderly patients. Delay in diagnosis and systemic conditions associated with advancing age probably contribute to poorer outcome from therapeutic measures.

(Br F Ophthalmol 2000;84:54-59)
\end{abstract}

Corneal infection is a leading cause of ocular morbidity and blindness worldwide. ${ }^{1-3}$ Trachoma alone affects more than 500 million people, of which six to nine million are blind. ${ }^{3}$ With the exception of one series ${ }^{4}$ recent reports on microbial keratitis have not discussed the elderly (defined as age $>65$ years),,$^{5-12}$ and there has been only one large report with a multicentre (Boston, Los Angeles, USA) patient series from 1977 to 1984 which specifically addresses microbial keratitis in the elderly in the USA. ${ }^{13}$ The more recent information is confined to only a very small series on elderly contact lens wearers. ${ }^{14}$ Consequently, there is a relative paucity of information on the prevalence and course of microbial keratitis in this age group.

In this study, the authors conducted an analysis of 102 cases of corneal ulceration in the elderly seen at a single tertiary eye care centre in urban southern India, with special attention paid to microbial aetiology, treatment modalities, and final outcome, both structural and visual.

\section{Patients and methods}

An analysis of information collected prospectively on a corneal ulcer database at the LV Prasad Eye Institute in Hyderabad, India, identified 102 cases of corneal ulceration in patients aged 65 years and older who were treated as inpatients for (non-viral) microbial keratitis between 1 February 1991 and 30 June 1995. The patients who met the following criteria were included in the study: (i) presence of corneal stromal infiltrate upon slit lamp examination; and (ii) microbiological evaluation of corneal scrapings for suspected microbial keratitis.

Initial examination was performed by various physicians over the course of the study, and the location and size of infiltrate were obtained by slit lamp examination using the length of the slit to measure the dimensions in patients who were able to cooperate for slit lamp biomicroscopic examination.

Using standard techniques, ${ }^{15}$ corneal scrapings, obtained by a sterile blade no 15 on Bard Parker handle, were inoculated directly onto sheep blood agar, chocolate agar, non-nutrient agar, Sabouraud's dextrose agar, potato dextrose agar, thioglycolate, and brain-heart infusion broth. Sabouraud's and potato dextrose agar plates were incubated at $25^{\circ} \mathrm{C}$ to enhance the growth of fungi, and the remainder were incubated at $37^{\circ} \mathrm{C}$. Blood agar plates were incubated under aerobic and anaerobic conditions, chocolate agar was incubated with 5\% carbon dioxide, and non-nutrient agar was incubated with an added Escherichia coli suspension. Gram stain, Giemsa stain, $\mathrm{KOH}$ preparation, and $\mathrm{KOH}$ with calcofluor white under fluorescence were included as part of the standard protocol for microscopic evaluation of corneal smears. Acid fast stains (ZiehlNeelsen and Kinyoun) were performed when indicated. A culture was considered positive when there was growth of the same organism on two or more media, confluent growth at site of inoculation on one solid medium, growth in one medium with consistent direct microscopy findings, or growth of the same organism on repeated corneal scraping. 


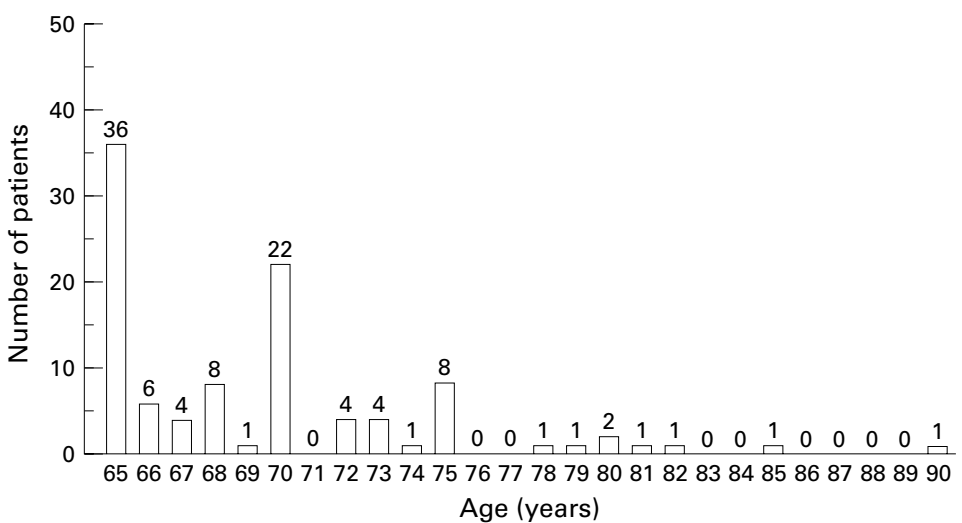

Figure 1 Age distribution of microbial keratitis in the elderly $(n=102)$ in southern India.

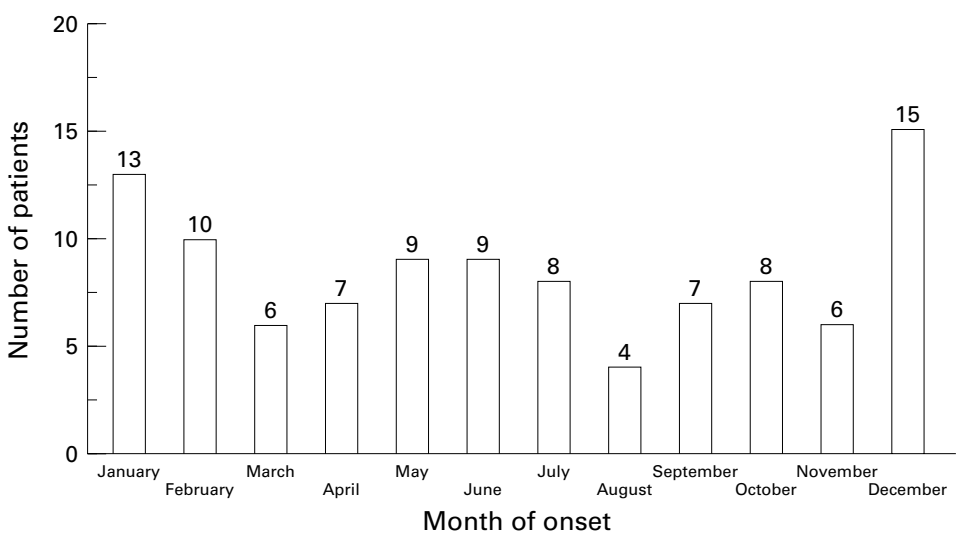

Figure 2 Seasonal variation in microbial keratitis in the elderly $(n=102)$ in southern India. bacitracin/polymixin B (Neosporin) eye ointment every 4 hours.

\section{Results}

DEMOGRAPHICS

A total of 102 patients met the inclusion criteria of this study, of which $72(70.6 \%)$ were male and $30(29.4 \%)$ were female. There were 47 left eyes and 55 right eyes involved. The average age of the patients was 69.3 (SD 5.0 years), with a range of 65-90 years. The age distribution is shown in Figure 1.

Monthly distribution of the cases is shown in Figure 2.

MICROBIOLOGICAL PROFILE

Seventy four $(72.5 \%)$ of the 102 cases were culture positive; in 19 cases (18.6\%), more than one organism was isolated. Seven $(25.0 \%)$ of the 28 culture negative patients and 25 (33.8\%) of the 74 culture positive patients had received previous antibiotic treatment. Organisms isolated from the culture positive group are shown in Table 1.

In 64 patients $(86.5 \%$ of 74 culture positive cases) one or more bacteria was isolated; in 19 patients $(25.7 \%)$, one or more fungi was isolated; and in one patient $(1.4 \%)$, Acanthamoeba species was isolated. Staphylococcus epidermidis (31.1\%), filamentous fungi (25.7\%), and Streptococcus pneumoniae (13.5\%)

Table 1 Organisms identified in cultures of corneal scrapings in the elderly

\begin{tabular}{|c|c|c|}
\hline Organisms & $\begin{array}{l}\text { Number of } \\
\text { isolates * } \\
(n=99)\end{array}$ & $\begin{array}{l}\text { Percentaget } \\
(n=74)\end{array}$ \\
\hline Total bacteria & & 86.5 \\
\hline Total Gram positive cocci & 46 & 62.2 \\
\hline Staphylococcus epidermidis & $23(7)$ & 31.1 \\
\hline Staphylococcus aureus & $8(4)$ & 10.8 \\
\hline Micrococcus species & 1 & 1.4 \\
\hline Streptococcus pneumoniae & $10(3)$ & 13.5 \\
\hline$\alpha$-haemolytic Streptococcus & $3(1)$ & 4.1 \\
\hline non-haemolytic Streptococcus & $1(1)$ & 1.4 \\
\hline Total Gram positive bacilli & 15 & 18.9 \\
\hline Corynebacterium species & $9(6)$ & 12.2 \\
\hline Corynebacterium pyogenes & $2(1)$ & 2.7 \\
\hline Corynebacterium hofmannii & $1(1)$ & 1.4 \\
\hline Bacillus species & 2 & 2.7 \\
\hline Bacillus subtilis & $1(1)$ & 1.4 \\
\hline \multicolumn{3}{|l|}{$\begin{array}{l}\text { Total Gram negative cocci } \\
\text { none }\end{array}$} \\
\hline Total Gram negative bacilli & 12 & 16.2 \\
\hline Pseudomonas aeruginosa & $8(3)$ & 10.8 \\
\hline Pseudomonas species & $2(1)$ & 2.7 \\
\hline Proteus mirabilis & $2(2)$ & 2.7 \\
\hline Moraxella species & 2 & 2.7 \\
\hline Moraxella lacunata & 1 & 1.4 \\
\hline unidentified anaerobic GNB & $1(1)$ & 1.4 \\
\hline Total parasites & & 1.4 \\
\hline Acanthamoeba species & $1(1)$ & 1.4 \\
\hline Total fungus & & 25.7 \\
\hline Total filamentous fungi & 21 & 25.7 \\
\hline Aspergillus flavus & 4 & 5.4 \\
\hline Aspergillus terreus & 1 & 1.4 \\
\hline Aspergillus niger & $1(1)$ & 1.4 \\
\hline Aspergillus species & 1 & 1.4 \\
\hline Curvularia lunata & $2(2)$ & 2.7 \\
\hline Curvularia verruculosa & 1 & 1.4 \\
\hline Exserohilum species & 1 & 1.4 \\
\hline Fusarium roseum & $2(2)$ & 2.7 \\
\hline Fusarium solani & $1(1)$ & 1.4 \\
\hline unidentified dematiaceous fungus & $5(3)$ & 6.8 \\
\hline unidentified hyaline fungus & $2(1)$ & 2.7 \\
\hline $\begin{array}{l}\text { Total yeast-like fungi } \\
\text { none }\end{array}$ & & \\
\hline
\end{tabular}

*Polymicrobial cultures in parentheses.

+Percentage of culture positive cases $(n=74)$. Total is greater than $100 \%$ because of polymicrobial infections.
TREATMENT

The standard treatment protocol was as follows.

Bacterial keratitis

Before 1995, fortified cefazolin (5.0\%) and gentamicin $(1.4 \%)$ every minute for 5 minutes and then every 30 minutes for the first day, to be tapered according to clinical response. From 1995, the standard initial treatment protocol by most ophthalmologists was topical ciprofloxacin $(0.3 \%)$ every minute for 5 minutes and then every 30 minutes for the first day, to be tapered according to clinical response. Fortified cefazolin and gentamicin were used if Streptococcus pneumoniae was suspected by smear studies.

Fungal keratitis

One drop natamycin suspension (5\%) every 5 minutes for 30 minutes and then every 30 minutes, to be tapered according to clinical response. Epithelium was debrided regularly to increase drug penetration. Oral ketoconazole at a dosage of $200 \mathrm{mg}$ twice daily was added when the ulcer was more than $6 \mathrm{~mm}$ in diameter, deeper than the anterior one half of the stroma, or if anterior chamber exudates were present. Some patients received a combination of topical clotrimazole $(1 \%)$, miconazole $(1 \%)$, and ketoconazole $(1 \%)$.

Acanthamoeba keratitis

Topical polyhexamethylene biguanide $(0.02 \%)$ eye drops every half hour with neomycin/ 
Table 2 Predisposing factors for microbial keratitis in the elderly

\begin{tabular}{lll}
\hline & $\begin{array}{l}\text { Number of } \\
\text { patients } \\
(n=102)\end{array}$ & $\begin{array}{l}\text { Prevalence } \\
\text { (\%) }\end{array}$ \\
\hline Predisposing factors & 39 & 38.2 \\
Ocular disease & 12 & \\
glaucoma & 7 & \\
corneal oedema & 6 & \\
corneal scarring & 4 & \\
previous ulcer, same eye & 4 & \\
dry eyes & 2 & \\
corneal degeneration & 1 & \\
trichiasis & 1 & \\
xerophthalmia & 1 & \\
conjunctivitis & 1 & \\
lagophthalmos & 30 & \\
Previous ocular surgery, same eye & 18 & 17.6 \\
Trauma & 5 & \\
plant & 2 & \\
dirt & 2 & \\
contact lens & 1 & \\
stone & 1 & \\
chemical & 7 & \\
not identified & 17 & \\
Systemic disease & 14 & \\
diabetes mellitus & 1 & \\
rheumatoid arthritis & 1 & \\
Stevens-Johnson syndrome & 1 & \\
measles & & \\
& &
\end{tabular}

^Total is greater than 102 because of multiple predisposing factors in some patients.

were the most common isolates. Gram positive cocci accounted for $62.2 \%$, fungi for $25.7 \%$, Gram negative bacilli for $21.6 \%$, Gram positive bacilli for $18.9 \%$, and parasites for $1.4 \%$ of culture positive cases. No Gram negative cocci and no yeast-like fungi were isolated in this population.

CLINICAL EXAMINATION

The location of corneal infiltrate was central in $53(52.0 \%$ ) eyes, peripheral (within $4 \mathrm{~mm}$ of the limbus) in $22(21.6 \%)$, and spanned both central and peripheral locations in $27(26.5 \%)$. The area of infiltrate was small $(<2 \mathrm{~mm}$ in greatest dimension) in $22(21.6 \%)$ eyes, medium (2-6 $\mathrm{mm}$ ) in 50 eyes $(49.0 \%)$, and large $(>6 \mathrm{~mm})$ in 30 eyes $(29.4 \%)$. The average duration of complaints before examination was 33.0 days (SD 98.5 days, range $0-700$ days).

PREDISPOSING FACTORS

The predominant predisposing factor for infectious keratitis in this study population was ocular disease, which was associated with $35.3 \%$ of cases. These included glaucoma in 12 patients, corneal oedema in seven, corneal

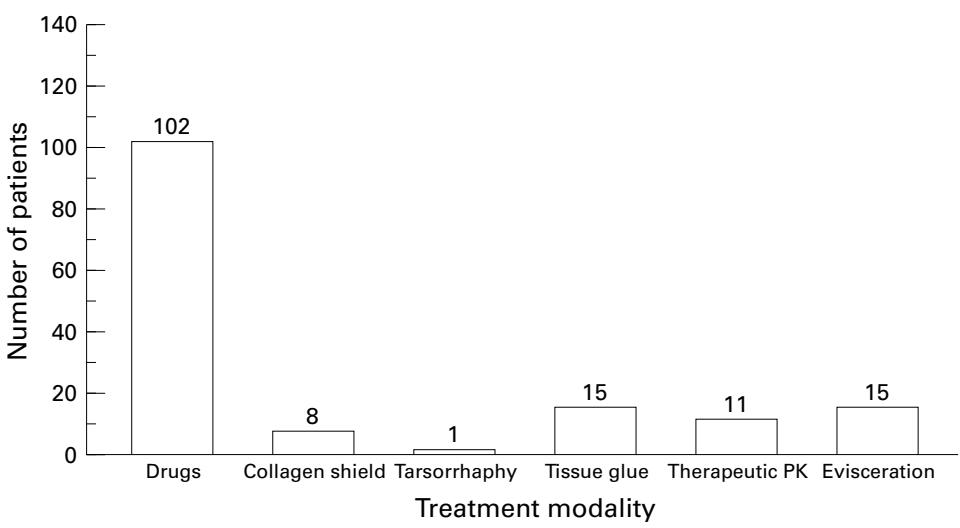

Figure 3 Treatment modality of the elderly $(n=102)$ with microbial keratitis in southern India. scarring in six, previous corneal ulcer in four, dry eyes in four, corneal degeneration in two, and trichiasis, xerophthalmia, conjunctivitis, and lagophthalmos in one patient each. Other predisposing factors included previous ocular surgery of any kind in the same eye $(29.4 \%)$, trauma (17.6\%), and severe systemic disease $(16.7 \%)$. Within the category of trauma, the causes were plant matter in five patients, stone in one patient, chemicals in one patient, and an unidentified source in seven patients. A history of contact lens use was present in two patients. Within the category of systemic disease, a history of diabetes mellitus was present in 14 patients, rheumatoid arthritis in one, Stevens-Johnson syndrome in one, and measles in one patient. The details are provided in Table 2 .

\section{TREATMENT PLAN AND OUTCOME}

All patients in the series were given antimicrobial medical therapy (see standard treatment in Methods). However, 11 patients $(10.8 \%)$ required therapeutic penetrating keratoplasty, $15(14.7 \%)$ required cyanoacrylate tissue adhesive and bandage contact lens application, eight $(7.8 \%)$ were treated with a collagen shield or bandage contact lens, one $(1.0 \%)$ underwent tarsorrhaphy, and 15 $(14.7 \%)$ eventually required evisceration. All patients who ultimately underwent evisceration presented with no light perception or inaccurate projection of light/rays, eight cases $(53.3 \%)$ presented with total corneal infiltrate, and five cases $(33.3 \%)$ presented with concurrent endophthalmitis. The pathogens involved with these evisceration cases included fungi in five cases $(33.3 \%$, two of which were polymicrobial), Pseudomonas aeruginosa in three cases $(20.0 \%$, one of which was polymicrobial), Streptococcus pneumoniae in three cases (20.0\%), Corynebacterium species in two cases $(13.3 \%$, both of which were polymicrobial), Staphylococcus epidermidis in one case $(6.7 \%)$, and no organism was isolated in two cases $(13.3 \%)$. Treatments are illustrated in Figure 3.

The predominant outcome was a corneal scar, with or without vascularisation (63 or $67.0 \%$ of the 94 followed cases) at last follow up, which ranged between 3 days and 4 years from the date of presentation (mean 26.5 (SD 55.5) weeks). Eight patients were lost to follow up. Other outcomes included evisceration (16.0\% of followed cases), adherent leucoma $(3.2 \%)$, clear graft $(2.1 \%)$, glaucoma $(1.1 \%)$, and anterior staphyloma (1.1\%) (Fig 4).

\section{VISUAL ACUITY AT LAST FOLLOW UP}

Visual acuity was recorded for 94 patients at last follow up, ranging between 3 days and 4 years from presentation date (mean 26.5 (55.5) weeks) (Fig 5). The remaining eight cases were lost to follow up. Nine $(9.6 \%)$ of the 94 followed patients achieved an unaided vision of 20/60 or better at last follow up, and $23(24.5 \%)$ achieved an unaided vision of $20 / 400$ or better at last follow up. 


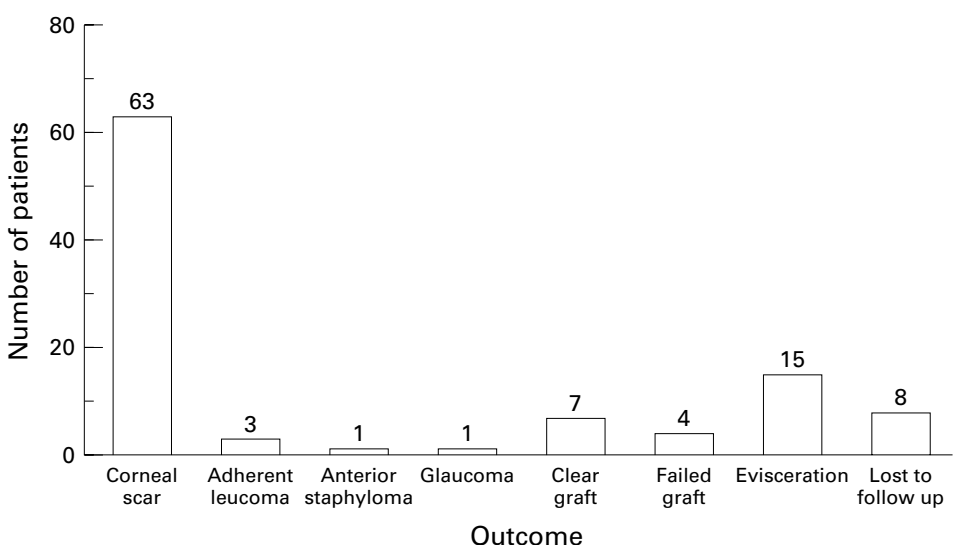

Figure 4 Outcome at last follow up in elderly patients $(n=102)$ with microbial keratitis in southern India.

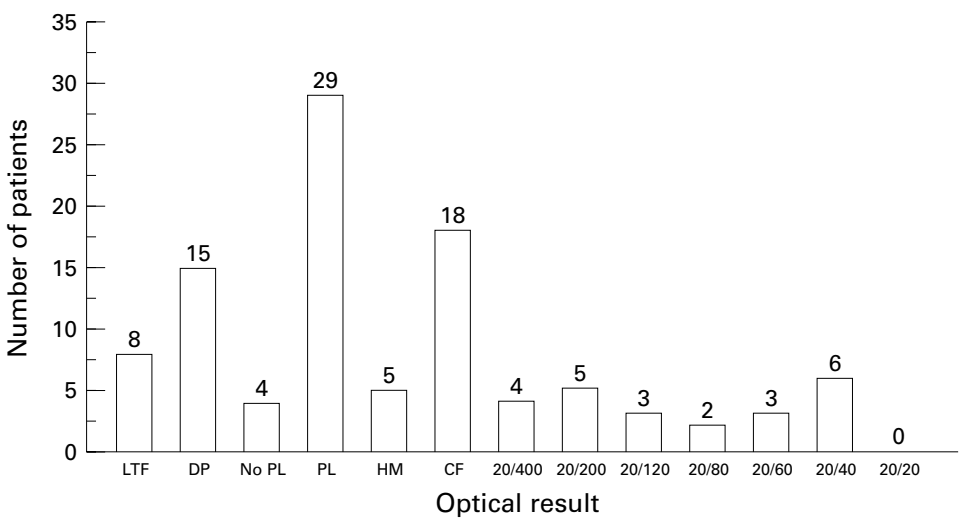

Figure 5 Optical result at last follow up for followed elderly patients $(n=102)$ with microbial keratitis in southern India.

\section{Discussion}

DEMOGRAPHICS

Male preponderance in this series was not different from the reported age independent microbial keratitis from the USA wherein $71 \%$ were males and $20 \%$ were females. ${ }^{4}$ However, compared with a series reported in children from this institute ${ }^{16}$ (males $56.1 \%$, females $43.9 \%$ ) and another age independent series from south India ${ }^{17}$ (males $61.3 \%$, females $38.7 \%$ ) the incidence of microbial keratitis in this series was significantly higher in males than females ( $p=<0.0001)$. Considering the predominant predisposing factors in this study population, the probable reasons for the male preponderance are unknown.

\section{MICROBIOLOGICAL PROFILE}

The prevalence of different organisms responsible for age independent microbial keratitis varies in different regions of the world. ${ }^{4-12}$ Indeed, the prevalence of organisms responsible for microbial keratitis in the elderly in this study likewise varies from a study on the elderly in the United States, ${ }^{13}$ as well as from other series on microbial keratitis..$^{4-12} 1819$

Despite the variation, with respect to bacterial keratitis, our series has certain similarities with the observations made by Ormerod ${ }^{13}$ who examined only bacterial keratitis in the elderly. Both series implicate Gram positive cocci as the leading pathogens of bacterial keratitis in the elderly, which account for $71.9 \%$ of bacte- rial cases in this series versus $71 \%$ in Ormerod's series. Both series also implicate Gram negative cocci/coccobacilli as the pathogens which cause the least percentage of bacterial keratitis in the elderly, accounting for $6.3 \%$ of bacterial cases in this series and $5 \%$ in Ormerod's series. ${ }^{13}$ However, there is a difference in the prevalence of Gram positive and Gram negative bacilli groups between the two series. Gram positive bacilli keratitis accounted for $23.4 \%$ in our series versus $1 \%$ in Ormerod's series, and Gram negative bacilli keratitis accounted for $18.8 \%$ in our series versus 39\% in Ormerod's series. ${ }^{13}$

The prevalence of Pseudomonas species were similar to the other study done on bacterial keratitis in the elderly. ${ }^{13}$ However, one distinction between the two series was that no Pseudomonas species keratitis was associated with contact lens wear in this series, unlike those of Ormerod $^{13}$ (one quarter of Pseudomonas keratitis was contact lens associated) and other series, ${ }^{511}$ which have commonly found Pseudomonas species keratitis associated with contact lens wear.

In this study, fungi were found to be responsible for $25.7 \%$ of microbial keratitis cases, all of which were caused by filamentous fungi. Although the study on the elderly by Ormerod $^{13}$ does not include fungal keratitis, rates in this series are consistent with those reported in age independent series from southern or tropical climates. ${ }^{57917}$ The high rate of filamentous fungi may be attributed to a higher rate of agriculturally based livelihood and trauma by organic matter in this part of the world. Prevalence rates of Candida species in this series is lower than those reported in other age independent series. ${ }^{1113}$

Also of note is the prevalence of Acanthamoeba keratitis in this series $(1.4 \%$ of culture positive cases), and the fact that the one Acanthamoeba infection in this series was not associated with contact lens use. In India, Acanthamoeba keratitis is responsible for about 3-5\% of all culture positive cases (Sharma S, unpublished data, 1997). This percentage is similar to rates reported in North America $(4.5 \%) .{ }^{20}$ Of the Acanthamoeba keratitides, only $4.5 \%$ are contact lens related on the Indian subcontinent ${ }^{21}$ compared with $85 \%$ reported in North America. ${ }^{22}$ This difference is probably due to a decreased prevalence of contact lens use in this part of the world.

The results of this series, suggest that Staphylococcus epidermidis (31.1\%), filamentous fungi $(25.7 \%)$, Corynebacterium species (16.3\%), Streptococcus pneumoniae (13.5\%), and Pseudomonas aeruginosa (13.5\%) are the leading aetiological microbes of microbial keratitis in the elderly.

It is of interest to note that the prevalence of these pathogens is similar to those reported in other series from southern or tropical climates, ${ }^{5} 7917$ and to a paediatric series (ages newborn to 16 years) from this eye institute, ${ }^{16}$ suggesting that corneal pathogens which afflict the elderly are related more to the local microbial flora than to a tropism for the elderly population. 
An attempt was made to seek association of size of the corneal infiltrate and duration of symptoms to that of culture positivity of the corneal scrapings, in this series. Larger ulcers and symptoms with longer duration may be expected to be associated with greater positivity in culture. However, no significant difference was found by $\chi^{2}$ test between culture positivity in ulcer size $<2 \mathrm{~mm}$ greatest dimension $(17 / 22,77.3 \%)$ and culture positivity in ulcers with $>6 \mathrm{~mm}$ greatest dimension $(23 / 30,76.7 \%), p=0.959$. Similarly, numbers of culture positive cases were not significantly greater in patients presenting with duration of symptoms more than 30 days ( $<30$ days, culture positive $64 / 84,76.2 \%) ;(\geqslant 30$ days, culture positive $10 / 18,55.6 \%) ; \mathrm{p}=0.075)$.

\section{PREDISPOSING FACTORS}

Traumatic corneal injury is the leading cause of age independent microbial keratitis. ${ }^{4}$ However, in this series of elderly patients, trauma was the third leading predisposing factor to microbial keratitis $(17.6 \%)$, following previous ocular disease $(35.3 \%)$ and previous ocular surgery in the same eye $(29.4 \%)$. Another series similarly found trauma to be a relatively less important predisposing factor to microbial keratitis in the elderly. ${ }^{13}$ Also of note is that only two cases $(2 \%)$ in this series were associated with contact lens use, whereas $12 \%$ in another elderly keratitis series were associated with soft lens use. ${ }^{13}$ This may be attributed to low usage of contact lenses in this part of the world.

Within the category of ocular disease glaucoma was an important predisposing factor, accounting for $30.8 \%$ of patients presenting with a history of previous ocular disease (Table 2). Keratitis associated with the use of glaucoma medication and, in particular, timolol has been well described in the literature. ${ }^{23-25}$ Several studies describe a significantly prolonged corneal anaesthesia effect with timolol use in an older age group. ${ }^{24}{ }^{25}$ In the present series, four $(33.3 \%)$ of the 12 elderly patients with glaucoma as a predisposing factor had a current history of timolol use. There was no reason to suspect drop contamination. Eight out of 12 patients $(66 \%)$ had end stage glaucoma before developing corneal ulceration. Notably, $13.7 \%$ of all patients in this series had associated diabetes mellitus (consistent with diabetes prevalence in the general Indian population, and thus not a likely independent predisposing factor), and $29.4 \%$ of all patients had previous ocular surgery of any kind in the same eye. These factors highlight a clinical picture typical of this population, that age related disease often accompanies microbial keratitis in the elderly.

TREATMENT PLAN AND OUTCOME

All eyes were given antimicrobial medication. Twelve eyes $(11.8 \%)$ required surgical intervention, including 11 penetrating keratoplasties and one tarsorrhaphy. Of the cases which required penetrating keratoplasties, seven grafts $(63.6 \%)$ remained clear. An active infectious process with severe inflammation and a large graft diameter due to a significant area of corneal involvement are factors that contributed to a slightly lower success rate. However, it should be pointed out that compared with paediatric populations which had clear graft rates ranging from $14-50 \%$ in other series, ${ }^{18}{ }^{19}$ the elderly population responds relatively well to penetrating keratoplasty.

Fifteen cases $(14.7 \%)$ ultimately required evisceration or enucleation secondary to being refractory to other treatment, a figure higher but similar to the $7 \%$ cited in another series on the elderly. ${ }^{13}$ These evisceration rates are higher than the $2 \%$ range reported in other series on the general population. ${ }^{12}$ Delay in presentation and consequent advanced nature of corneal infection may be partly responsible for this higher rate of evisceration. Other outcomes of the microbial keratitis in the elderly episode included corneal scarring, adherent leucoma, glaucoma, anterior staphyloma, and a disorganised globe.

Visual acuity was recorded in 94 cases, the remainder being lost to follow up. The optical results of the microbial keratitis event in Figure 5 show that a very poor visual acuity was achieved. The authors feel that the main reason for this is a problem rooted in the psyche of the elderly patient. Owing to physical dependency, all too often an elderly patient will wait for long periods of time before seeking medical attention, especially those depending on the relatives to accompany them. In this series, some patients reported subjective ocular complaints for nearly 2 years before seeking treatment. The average duration of ocular complaints in this series was 33.05 days (SD 98.99 days, median 9 days, range $0-700$ days). This is higher than that observed in a childhood series from the same institution (mean 14.42 days, SD 32.34 days, median 6 days, range 0-180 days) in patients aged newborn to 16 years. ${ }^{16} \mathrm{It}$ is important to educate both elderly patients and their families as to when they should seek medical aid with attention paid to the appropriate predisposing factors, and to schedule annual eye examinations where possible to ensure such complaints are at least addressed yearly.

This study is limited by vague inclusion criteria, a problem difficult to obviate owing to a lack of any gold standard for what constitutes a microbial keratitis. A study on microbial keratitis should of course ideally include only keratitis which is microbial in aetiology, but even under the best of conditions, a causative microbe can be isolated in only about $40-80 \%$ of cases, ${ }^{4-121819}$ a rate decreased further if the patient has received previous antibiotic treatment from a referring ophthalmologist or general practitioner.

Finally, although it may be difficult in a small, private clinical practice to follow the proper protocol of taking corneal scrapings in all suspected cases, and modifying treatment based on microbiology reports, it is none the less essential. Although for a small lesion, not above the visual axis and not suggestive of fungal or viral aetiology, treatment with broad spectrum antibiotics with close follow up has 
been suggested ${ }^{26}$ without microbiological investigation, the same cannot be applied in tropical climates where fungal keratitis is an enormous problem. ${ }^{17}$

In summary, this study represents the largest single centre series on microbial keratitis in the elderly. Microbial pathogens responsible for keratitis in the elderly appear to be driven by the local microbial flora rather than by a predilection for elderly patients, and the predisposing factors differ from those of the general population with previous ocular disease and surgery as the leading factors. Medical treatment and surgical intervention rates are similar to the general population, but a higher evisceration rate is seen in the elderly population. Visual acuity results tend to be poor, best explained by a delayed seeking of eye care. With the information presented in this paper, it is hoped that clinicians will assist in increasing health awareness in their elderly patients, and that proper identification of predisposing factors, aetiological microbes, and treatment outcomes will aid in the better diagnosis and management of microbial keratitis in the elderly.

Financial support: Hyderabad Eye Research Foundation. Proprietary interest: none.

1 Burd EM, Ogawa GSH, Hyndiuk RA. Bacterial keratitis and conjunctivitis. In: Smolin G, Thoft RA, eds. The cornea. Scientific foundations and clinical practice. 3rd ed. Bornea. Scientific foundations and Little, Brown, 1994:115-67.

2 Abiose A. Update: onchocerciasis. In: Kupfer C, Gillen T, eds. World blindness and its prevention. Volume 4. International Agency for the Prevention of Blindness, 1990: national

3 Adela, HS. Organization of a trachoma-control program in Africa, with special emphasis on Kenya. In: Kupfer C, Gillen $\mathrm{T}$, eds. World blindness and its prevention. Volume 4 International Agency for the Prevention of Blindness, 1990:193-7.

4 Ormerod LD, Hertzmark E, Gomez DS, et al. Epidemiology analysis. Ophthalmology 1987;94:1322-33.
5 Liesegang TJ, Forster RK. Spectrum of microbial keratitis in south Florida. Am f Ophthalmol 1980;90:38-47.

6 Asbell P, Stenson S. Ulcerative keratitis: survey of 30 years' laboratory experience. Arch Ophthalmol 1982;100:77-80.

7 Gonawardena SA, Ranasinghe KP, Arseculratne SN, et al. Survey of mycotic and bacterial keratitis in Sri Lanka. Mycopathologia 1994;127:77-81.

8 Neumann M, Sjostrand J. Central microbial keratitis in a Swedish city population. A three-year prospective study in Gothenburg. Acta Ophthalmol Copenh 1993;71:160-4

9 Williams G, McClellan K, Billson F. Suppurative keratitis in rural Bangladesh: the value of gram stain in planning management. Int Ophthalmol 1991;15:131-5.

10 Wahl JC, Katz HR, Abrams DA. Infectious keratitis in Baltimore. Ann Ophthalmol 1991;23:234-7.

11 McClellan KA, Bernard BJ, Billson FA. Microbial investigations in keratitis at the Sydney Eye Hospital. Aust NZ F Ophthalmol 1989;17:413-16.

12 Ormerod LD. Causation and management of microbial keratitis in subtropical Africa. Ophthalmology 1987;94: $1662-8$.

13 Ormerod LD. Causes and management of bacterial keratitis in the elderly. Can $\mathcal{F}$ Ophthalmol 1989;24:112-16.

14 Lemp MA, Blackman HJ, Wilson LA, et al. Gram-negative corneal ulcers in elderly aphakic eyes with extended-wear lenses. Ophthalmology 1984;91:60-3.

15 Jones DB, Liesegang TJ, Robinson NM. Laboratory diagnosis of ocular infections. Washington DC: Cumitech 13, American Society for Microbiology, 1981.

16 Kunimoto DY, Reddy MK, Sharma S, et al. Microbial keratitis in children. Ophthalmology 1998;105:252-7.

17 Srinivasan M, Gonzales CA, George C, et al. Epidemiology and aetiological diagnosis of corneal ulceration in Madurai, south India. Br f Ophthalmol 1997;81:965-71.

18 Ormerod LD, Murphree AL, Gomez DS, et al. Microbial keratitis in children. Ophthalmology 1986;94:449-55.

19 Cruz OA, Sabir AM, Capo H, et al. Microbial keratitis in childhood. Ophthalmology 1993;100:192-6.

20 Laibson, PR, Cohen EJ, Rajpal RK. Corneal ulcers related to contact lenses. CLAO $\mathcal{F} 1993 ; 19: 73-8$.

21 Stehr-Green JK, Bailey TM, Visvesvara GS. The epidemiology of Acanthamoeba keratitis in the United States. Am $\mathcal{F}$ Ophthalmol 1989;107:331-6.

22 Agrawal V, Biswas J, Madhavan HN, et al. Current perspectives in infectious keratitis. Indian $\mathcal{F}$ Ophthalmol 1994;42: 171-91

23 Van Buskirk EM. Adverse reactions from timolol administration. Ophthalmology 1980;87:447-50.

24 Van Buskirk EM. Corneal anesthesia after timolol maleate therapy. Am f Ophthalmol 1979;88:739-43.

25 Weissman SS, Asbell PA. Effects of topical timolol (0.5\%) and betaxolol $(0.5 \%)$ on corneal sensitivity. Br f Ophthalmol 1990;74:409-12.

26 McDonnell PJ. Empirical or culture-guided therapy for microbial keratitis? A plea for data. Arch Ophthalmol 1996; 114:84-7 